\title{
A Feature Extractor IC for Acoustic Emission Non-destructive Testing
}

\author{
Daniele Giardino $^{\# 1}$, Marco Matta ${ }^{\# 2}$, Sergio Spanò ${ }^{\# 3}$ \\ \# Department of Electronic Engineering, University of Rome Tor Vergata, Via Del Politecnico 1, Rome, 00133, Italy \\ E-mail: ${ }^{1}$ giardino@ing.uniroma2.it; ${ }^{2}$ matta@ing.uniroma2.it; ${ }^{3}$ spano@ing.uniroma2.it
}

\begin{abstract}
In this paper, we present the design and the implementation of a digital Application Specific Integrated Circuit (ASIC) for Acoustic Emission (AE) non-destructive testing. The AE non-destructive testing method is a diagnostic method used to detect faults in mechanically loaded structures and components. If a structure is subjected to mechanical load or stress, the presence of structural discontinuities releases energy in the form of acoustic emissions through the constituting material. The analysis of these acoustic emissions can be used to determine the presence of faults in several structures. The proposed circuit has been designed for IoT (Internet of Things) applications, and it can be used to simplify the existing procedures adopted for structural integrity verifications of pressurized metal tanks that, in some countries, they are based on periodic checks. The proposed ASIC is provided of Digital Signal Processing (DSP) capabilities for the extraction of the main four parameters used in the AE analysis that are the energy of the signal, the duration of the event, the number of the crossing of a certain threshold and finally the maximum value reached by the AE signal. The circuit is provided of an SPI interface capable of sending and receiving data to/from wireless transceivers to share information on the web. The DSP circuit has been coded in VHDL and synthesized in $90 \mathrm{~nm}$ technology using Synopsys. The circuit has been characterized in terms of area, speed, and power consumption. Experimental results show that the proposed circuit presents very low power consumption properties and low area requirements.
\end{abstract}

Keywords - IoT; acoustic emission; non-destructive testing; DSP; ASIC.

\section{INTRODUCTION}

Internet of Things (IoT) consists of smart devices equipped in the object, people, animals, and structures that can communicate over the Internet. IoT favors the implementation of several applications such as health care [1]-[3], smart cities [4], emergency systems [5], localization [6], gaming, UAV [7], agriculture, animal monitoring [8],[9] and other fields [10]-[22]. The Acoustic Emission (AE) method is a commonly applied Non-Destructive (ND) technique used to detect faults in mechanically loaded structures and components. If a structure is subjected to mechanical load or stress, the presence of structural discontinuities releases energy in the form of acoustic emissions through the constituting material. The AE method allows checking the integrity of a wide variety of structures by analyzing data coming from piezoelectric sensors.

One of the most common applications in this field is the testing of the structural integrity of pressure tanks. In some countries (for example Italy), the current legislation provides for the use of this technique. The actual AE method protocol is based on periodic checks that do not allow continuous monitoring and make use of very unwieldy instrumentation (Fig. 1). AE refers to the generation of transient elastic waves (Fig. 2) by a sudden redistribution of stress in a material [23], [24]. The analysis of the AE waves can be used to detect damage on structures. This method uses specific piezoelectric sensors for AE detection and electronic equipment for the signal analysis.

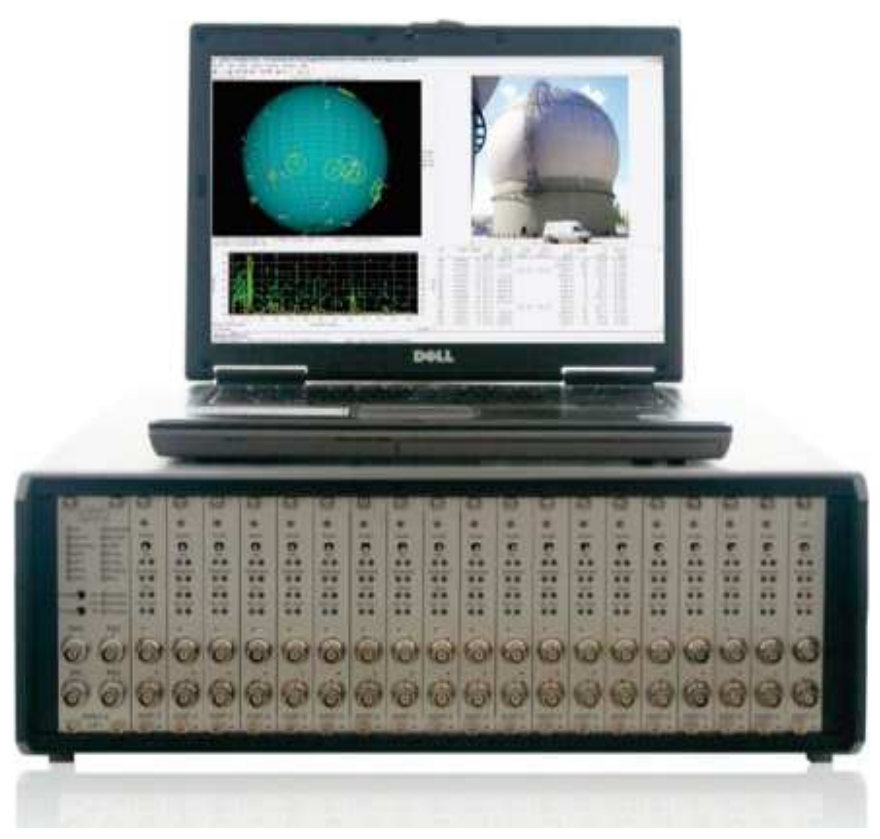

Fig. 1: Acoustic Emission instrumentation 


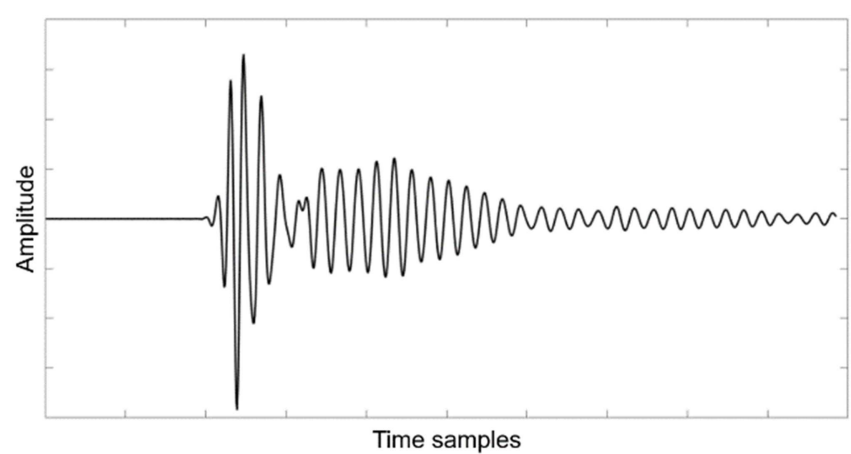

Fig.2: Acoustic Emission signal

The AE non-destructive testing method is performed analyzing some features of AE waves [23], [24]:

- the maximum of the absolute value of the amplitude;

- the signal duration;

- the signal energy;

- the number of crossings of a given threshold.

As introduced above, the present AE testing protocols are based on periodic manual checks.

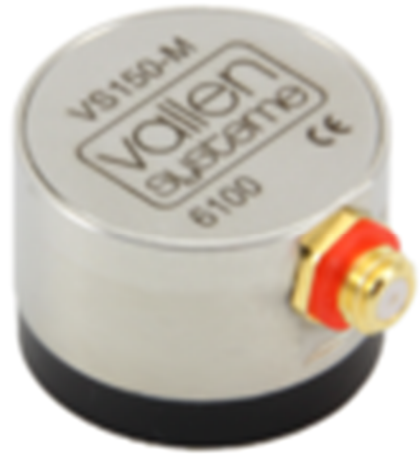

Fig.3: Acoustic Emission sensor

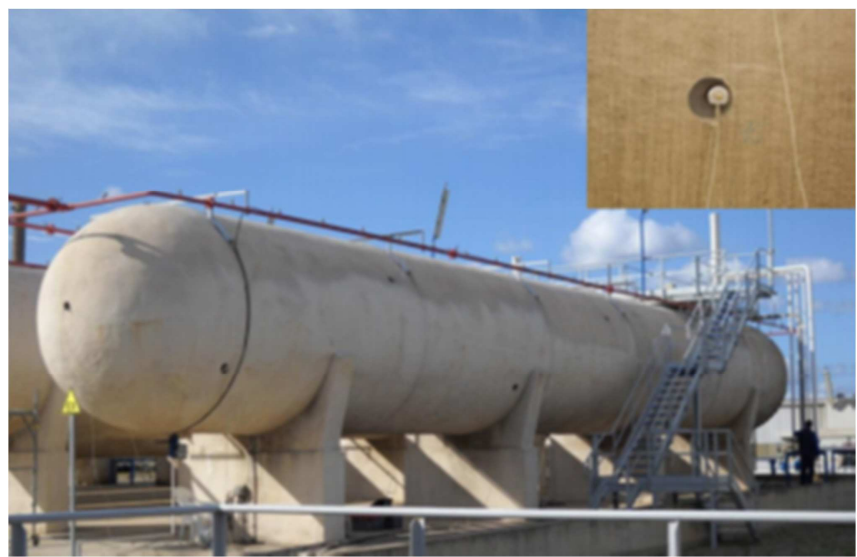

Fig.4 Pressure tank

During the periodic checks, AE sensors (Fig. 3) are installed on the structure under test by specialized operators (Fig. 4). These sensors are wired and connected with the equipment (Fig.1) that analyses data are coming from different sensors in terms of max amplitude, duration, energy, and several threshold crossings. This approach presents two main limitations:
- The real-time monitoring is not possible, and consequently, the health state of the structure is known only during the checks.

- The current procedures require very unwieldy instrumentation.

For such reasons, the introduction of the IoT technologies in the Acoustic Emission procedure is seen as an interesting solution because it is capable of allowing real-time monitoring.

In this context, power consumption and communication channel access are crucial aspects. In a wireless channel access scheme, some critical aspects must be considered. First, the access management of a large number of devices is trying to access the access point [24] - [27]. Secondly, the exploitation of licensed electromagnetic spectrum portions (already assigned to other systems) in an opportunistic way [28-32] nevertheless in the considered application the number of IoT sensors is limited.

Using the IoT approach, the sensor will always be connected to the Internet, and real-time monitoring would be assured. To replace the present sensors with IoT sensor nodes, an important issue is the minimization of power requirements [33] - [35]. Power consumption reduction can be obtained in different ways:

- Using new technologies, in terms of devices and materials [36-38].

- Using environment aware duty cycle optimization techniques [39]

- Using mixed HW/SW solutions. For example, using hardware accelerators to reduce the computation time and consequently the energy consumption [40] - [43]

- Using design techniques at RLT level as, for example, clock gating, clock enabling or approximated operators [34], [44]

- Using techniques at the layout level as, for example, power gating [34].

Batteries or energy harvesting sources usually power IoTot nodes. This is a limitation and implies that IoT nodes cannot make use of high-speed wireless communication protocols, as the high data rates lead to high power consumption. For this reason, the node must be provided of DSP (Digital Signal Processing) capabilities to process data coming from the piezoelectric sensor. As a consequence, it can send only the AE signal features that are useful for potential damage detection and analysis through the Internet.

In recent years, such IoT sensors nodes became popular also because of the increased interest and development of Machine Learning (ML) based low-power hardware accelerators [44] - [49]. Standard ML approaches require the design of a human-driven feature extraction model to produce appropriate data for the artificial intelligence learning process [50], [51].

The identification of an AE event can be performed using deterministic approaches or statistical ones. In most of the cases, a human operator is in charge of determining the nature of the $\mathrm{AE}$ triggered event, whether it is real damage or a false-positive. Given the circumstances of AE nondestructive testing, we automatized the feature extraction process for the automatic classifications of the incoming 
signals. In this paper, we present the hardware implementation of the DSP circuits of a wireless sensor node for IoT. Such courses have been implemented in $90 \mathrm{~nm}$ technology and characterized in terms of area, speed, and power consumption.

\section{MATERIAL AND METHODS}

As discussed in the previous section, the goal of this work is the hardware design of a digital circuit that can:

- Estimate the features useful for the Acoustic Emission method.

- Send the extracted data to a wireless transceiver. 5 .

The features required for the AE method are shown in Fig.

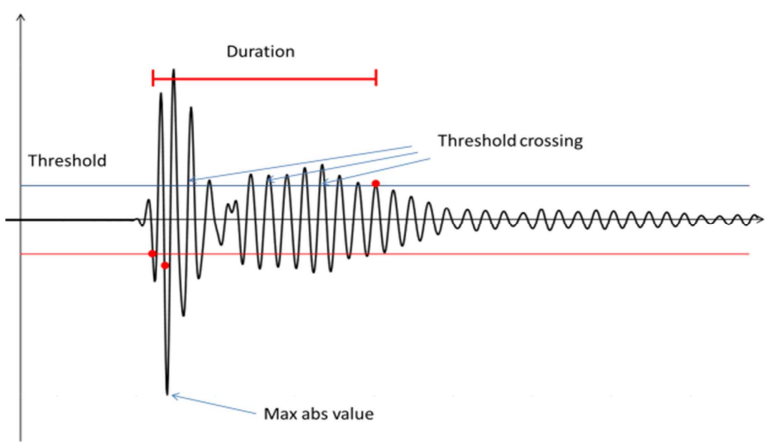

Fig.5: AE signal features

In the following subsections, we analyze the algorithms and the circuit used for the features estimation and the communication interface among the DSP circuit and the wireless transceiver.

\section{A. Duration}

The Duration of an AE signal is defined as the time interval between a start time and a stop time. The start-time corresponds to the moment in which the module of the signal is above a certain threshold. The stop-time is the instant when the signal goes below the threshold and maintains a lower value for at least $200 \mu \mathrm{s}$. This time is called DDT.

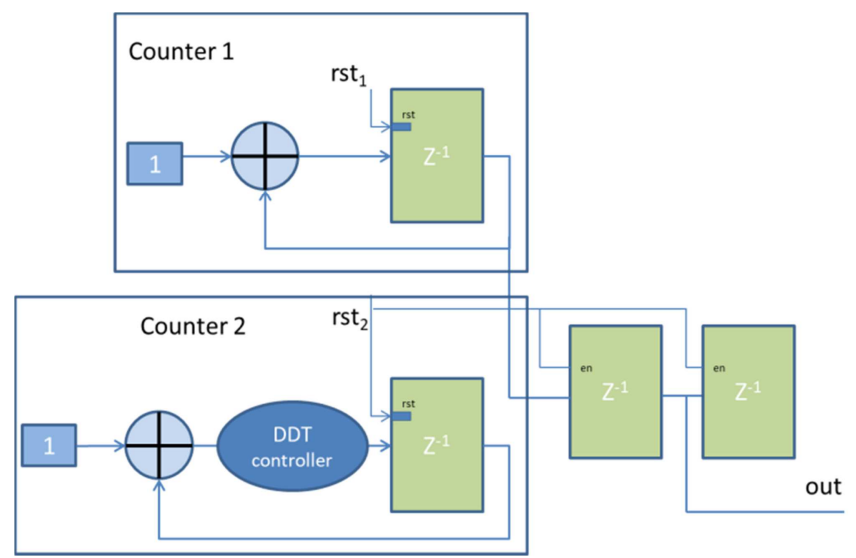

Fig.6 Circuit for the duration estimation

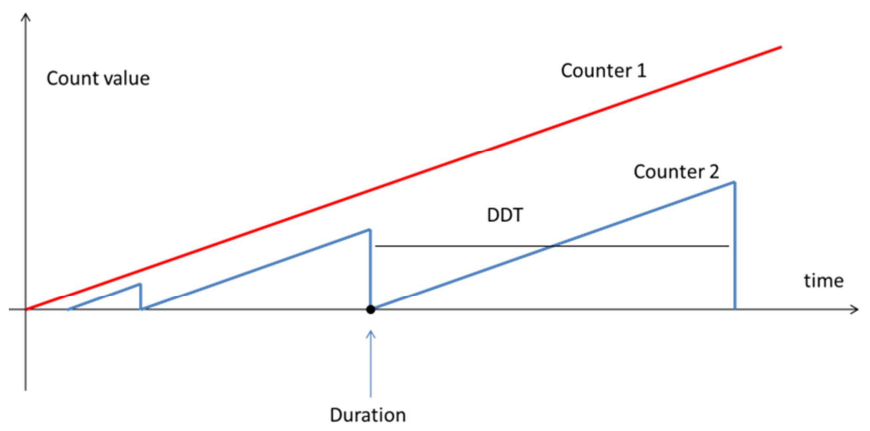

Fig. 7 Duration estimation

The duration estimator circuit has been implemented by two counters and a control logic net (Fig 6). The computational process of such an operation is shown in Fig. 7: when the absolute value of the signal exceeds a given threshold, the first counter (Counter 1) start to count. From this moment on, every time the signal goes below the threshold a second counter is reset, and it starts to count. This second counter is designed to count until $200 \mu$ s (the DDT time). If this counter reaches the DDT, the event is considered finished, and its duration corresponds to the previous second counter reset time. Vice-versa the second counter restart. Figure 8 shows the duration estimation circuits, composed of the two counters and the additional control logic.

\section{B. Number of threshold crossings}

The number of threshold crossings is defined as the number of times that the positive part of the signal goes across (below to above) the threshold, as shown in Fig. 5. This feature is estimated using the circuit shown in Fig. 8, which is composed of two delay blocks, two comparators, and a counter.

The circuit works as follows: the present and the previous values of the signal are respectively at the input and the output of the upper delay. Such values are compared with the threshold. If the signal goes across the threshold, the two comparators output is respectively 1 and 0 . When the crossing event occurs, the AND logic gate (with a negated input) provides 1 to the enable input of the counter that increments its value that corresponds to the number of threshold crossings.

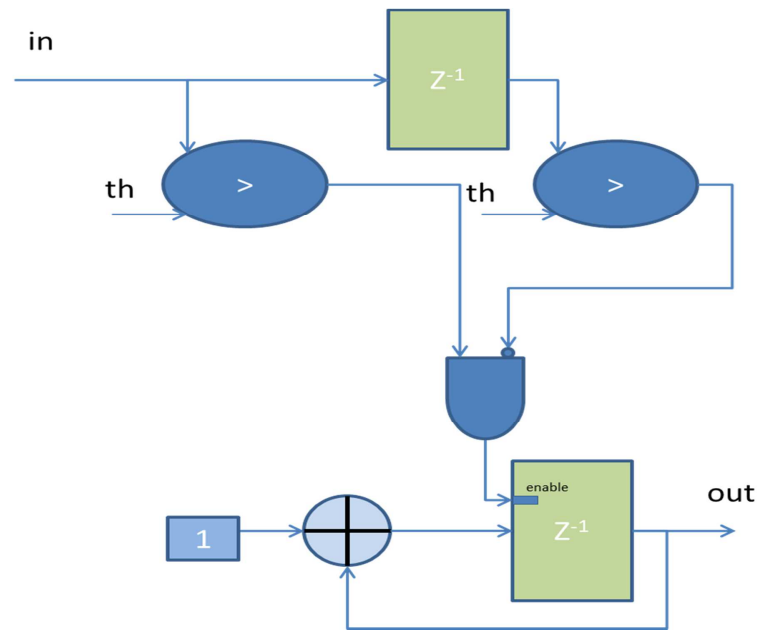

Fig. 8: Circuit for the number of crossings counting 


\section{Max Value}

The maximum of the absolute value of the $\mathrm{AE}$ signal is another signal feature required for the AE diagnostic method. This value is estimated using the circuit shown in Fig. 9. The circuit is composed by a comparator, a delay, a multiplexer, and an absolute value net. The absolute value of the present value of the input signal is compared with the value stored in the delay that represents the present maximum (the comparator performs this operation). If the sample at the input is greater than the previous max value stored in the delay block, the newer sample becomes the new AE signal maximum.

\section{Energy}

The energy of the AE signal is defined in Eq.1

$$
E=\sum_{\text {start_time }}^{\text {stop_time }}|x[n]|^{2}
$$

The energy estimator has been implemented using a digital integrator and a multiplier for the power of two operations. The energy is computed in the time interval defined by the start time and the stop-time of the AE signal.

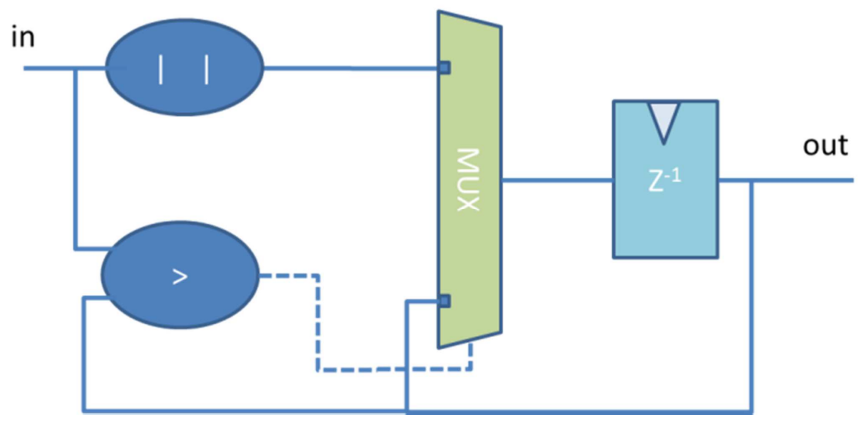

Fig. 9: Circuit for the maximum estimation

\section{E. IO Interface}

To communicate with other devices, the proposed circuits have been equipped with an IO interface. For this purpose, an SPI interface has been chosen. The communication procedure works as follows: when the DSP circuit ends the AE signal processing, a ready signal is asserted, and after one clock cycles the features are sent to the transceiver using the SPI protocol.

\section{RESULT AND DISCUSSION}

After a fixed-point optimization performed on MATLAB/ Simulink, the above-discussed system has been coded in VHDL (VSIC Hardware Description Language) at RTL abstraction level and then synthesized. The synthesis was performed by Synopsys Design Compiler using the STM 90 nm library of standard cells. Based on the results of the MATLAB/Simulink simulation, the system input and output dynamic range are 10 bits. Considering the narrow bandwidth of AE sensors used for non-destructive diagnosis, the clock frequency constraint was set to $5 \mathrm{MHz}$.
TABLE I

IMPLEMENTATION RESULTS

\begin{tabular}{|c|c|}
\hline Total cell area & $10508.8 \mu \mathrm{m}^{2}$ \\
\hline Clk Freq. & $5 \mathrm{MHz}$ \\
\hline Total Dynamic Power & $35.3 \mu \mathrm{W}$ \\
\hline Cell Leakage Power & $1.2 \mu \mathrm{W}$ \\
\hline Total Power consumption & $36,5 \mu \mathrm{W}$ \\
\hline
\end{tabular}

The power consumption analysis (at $5 \mathrm{MHz}$ ) shows that the cell leakage power is $1.2 \mu \mathrm{W}$ against the $35.5 \mu \mathrm{W}$ relatives to the total dynamic power. Being the Cell leakage power only $3.4 \%$ of the total Power consumption, the employment of Power gating to further reduce power consumption would not be justified. This is because, as shown in Fig 10, the power gating introduces a sizeable overhead (sleep transistors, isolation gates and extra routing for the sleep and isolate control signals as shown in [34]). Considering the results shown in Tab. 1, such additional complexity is not justified.

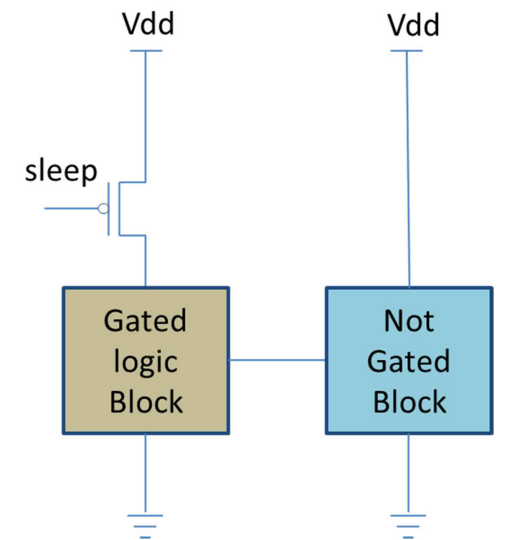

Fig. 10 Power gating by sleep transistor insertion

\section{CONCLUSIONS}

In this paper, we presented a DSP STM 90nm ASIC circuit of an IoT node designed for Acoustic Emission analysis. The proposed circuit can extract the features needed for the AE diagnostic method from the AE signal. The results show a much reduced area and limited power consumption. The total power consumption during the computation is only $35.3 \mu \mathrm{W}$. The system is feasible for its use in IoT-based sensors environment approaches. The sensors will always be connected to the Internet and the realtime monitoring is assured.

\section{REFERENCES}

[1] Scarpato N., Pieroni A., Di Nunzio L., Fallucchi F.. E-health-IoT universe: A review, 2017 International Journal on Advanced Science, Engineering and Information Technology 7(6), pp. 23282336

[2] H. Alemdar and C. Ersoy, Wireless sensor networks for healthcare: A survey Comput. Netw., vol. 54, no. 15, pp. 26882710, Oct. 2010.

[3] Islam, S.M.R., Kwak, D., Kabir, M.H., Hossain, M., Kwak, K.-S.The internet of things for health care: A comprehensive survey (2015) IEEE Access, 3, art. no. 7113786, pp. 678-708

[4] Pieroni, A., Scarpato, N., Di Nunzio, L., Fallucchi, F., \& Raso, M. Smarter city: Smart energy grid based on blockchain technology. 2018 International Journal on Advanced Science, Engineering and $\begin{array}{llr}\text { Information } & \text { Technology, } & 8(1),\end{array}$ doi:10.18517/ijaseit.8.1.4954

[5] L. Bracciale, P. Loreti, M. Luglio, C. Roseti, F. Zampognaro, SMARTSOS: Systems for maritime advanced rescue through satellite and optimized sensor networks (2017) Proceedings of the 2016 3rd 
International Conference on Information and Communication Technologies for Disaster Management, ICT-DM 2016

[6] Giuliano, R., Mazzenga, F., Petracca, M., \& Vari, M. (2013). Indoor localization system for first responders in emergency scenario. Paper presented at the 2013 9th International Wireless Communications and Mobile Computing Conference, IWCMC 2013, 1821-1826

[7] P. Loreti, P. Sperandio, M. Vettorello, M. Baldasseroni, A multitechnology indoor positioning service to enable new location-aware applications, (2012) Proceedings - 2012 IEEE 1st AESS European Conference on Satellite Telecommunications, ESTEL 2012

[8] Dalmasso, I., Galletti, I., Giuliano, R., \& Mazzenga, F. (2012). WiMAX networks for emergency management based on UAVs. Paper presented at the Proceedings - 2012 IEEE 1st AESS European Conference on Satellite Telecommunications, ESTEL 2012

[9] L. Bracciale, A. Catini, G. Gentile, P. Loreti, Delay tolerant wireless sensor network for animal monitoring: The Pink Iguana case, (2017) Lecture Notes in Electrical Engineering, 429, pp. 18-26.

[10] P. Loreti, A. Catini, M. De Luca, L. Bracciale, G. Gentile and C. Di Natale, (2019). The Design of an Energy Harvesting Wireless Sensor Node for Tracking Pink Iguanas. Sensors, 19(5), 985.

[11] Jipson George, Mohammed Nazeh Challenges Faced by CIOs in cloud and IoT based organizations- A Study on IT and Business Leaders International Journal on Informatics Visualization Vol 3, No 1 (2019)

[12] Sathyanarayanan PSV A Cloud Based Irrigation System for Agriculture International Journal on Informatics Visualization Vol 2, No 1 (2018)

[13] Mojtaba Jamshidi, Abdusalam Abdulla Shaltooki, Zahra Dagal Zadeh, Aso Mohammad Darwesh A Dynamic ID Assignment Mechanism to Defend Against Node Replication Attack in Static Wireless Sensor Networks International Journal on Informatics Visualization Vol 3, No 1 (2019)

[14] Vijaya Perumalla, B. Seetha Ramanjaneyulu, Ashok Kolli Simulation Study of Topological Structures and Node Coordinations for Deterministic WSN with $\mathrm{TSCH}$ International Journal on Informatics Visualization Vol 1, No 4 (2017)

[15] Gandeva Bayu Satrya, Haftu Tasew Reda,Kim Jin Woo, Philip Tobianto Daely, Soo Young Shin and Seog Chae, IoT and Public Weather Data Based Monitoring \& Control Software Development for Variable Color Temperature LED Street Lights, International Journal on Advanced Science, Engineering and Information Technology, vol. 7, no. 2, pp. 366-372, 2017.

[16] J. Ko, C. Lu, M. B. Srivastava, J. A. Stankovic, A. Terzis, and M. Welsh, Wireless sensor networks for healthcare,' Proc. IEEE, vol. 98, no. 11 , pp. 19471960 , Nov. 2010.

[17] Victor Rigworo Kebande,Nickson Karie Menza and H S Venter, Functional Requirements for Adding Digital Forensic Readiness as a Security Component in IoT Environments, International Journal on Advanced Science, Engineering and Information Technology, vol. 8, no. 2, pp. 342-349, 2018

[18] Jipson George Thoomkuzhy, Mohammed Nazeh Cyber Security and Information Assurance A Pa radigm Shift on the role of CIO's in Cloud and IOT based Organizations International Journal on Informatics Visualization Vol 2, No 4-2 (2018)

[19] Victor R Kebande, Nickson M Karie and H S Venter, "Adding Digital Forensic Readiness as a Security Component to the IoT Domain," International Journal on Advanced Science, Engineering and Information Technology, vol. 8, no. 1, pp. 1-11, 2018

[20] Ummi Wahida Badarudin,Wan Isni Sofiah Wan Din, Yuli Adam Prasetyo, Zalili Musa and Shahreen Kasim, Internet of Things: An Implementation and Its Challenges in Malaysia, International Journal on Advanced Science, Engineering and Information Technology, vol. 8, no. 6, pp. 2641-2647, 2018

[21] Jung Yeon Seo,Dae Won Lee and Hwa Min Lee, Performance Comparison of CRUD Operations in IoT based Big Data Computing, International Journal on Advanced Science, Engineering and Information Technology, vol. 7, no. 5, pp. 1765-1770, 2017

[22] Wenquan Jin, DoHyeun Kim A Sleep-Awake Scheme Based on CoAP for Energy-Efficiency in Internet of Things International Journal on Informatics Visualization Vol 1, No 4 (2017)

[23] Cardarilli G.C., Di Nunzio L, Massimi F, Fazzolari R, De Petris C, Augugliaro G, and Mennuti C. A wireless sensor node for Acoustic Emission non-destructive testing 2018 Lecture Notes in Electrical Engineering

[24] Eric Bechhoefer, Yongzhi Qu, Junda Zhu and David He:Signal Processing Techniques to Improve an Acoustic Emissions Sensor.
Proceedings of the Annual Conference of the Prognostics and Health Management Society 2013 pp. 581-58

[25] Giuliano, R., Mazzenga, F., Neri, A., \& Vegni, A. M. . Security access protocols in IoT capillary networks. 2017 IEEE Internet of Things Journal, 4(3), 645-657

[26] Ijaz, A., Zhang, L., Grau, M., Mohamed, A., Vural, S., Quddus, A. U., Tafazolli , R. (2016). Enabling massive IoT in 5G and beyond systems: PHY radio frame design considerations. IEEE Access, 4, 3322-3339.

[27] Jiang, N., Deng, Y., Kang, X., \& Nallanathan, A. (2018). Random access analysis for massive IoT networks under a new spatiotemporal model: A stochastic geometry approach. IEEE Transactions on Communications, 66(11), 5788-5803

[28] Mazzenga, F., Giuliano, R., \& Vatalaro, F. (2017). FttC-based fronthaul for $5 G$ dense/ultra-dense access network: Performance and costs in realistic scenarios. Future Internet, 9(4)

[29] Petracca, M., Pomposini, R., Mazzenga, F., Giuliano, R., \& Vari, M. (2010). An always available control channel for cooperative sensing in cognitive radio networks. Paper presented at the 2010 IFIP Wireless Days, WD 2010, doi:10.1109/WD.2010.5657704

[30] Petracca, M., Mazzenga, F., Pomposini, R., Vatalaro, F., \& Giuliano, R. (2011). Opportunistic spectrum access based on underlay UWB signalling. Paper presented at the Proceedings - IEEE International Conference on Ultra-Wideband, 180-184.

[31] L. Bracciale, P. Loreti, G. Bianchi, Human time-scale duty cycle for opportunistic WiFi based mobile networks (2013) 24th Tyrrhenian International Workshop on Digital Communications - Green ICT, TIWDC 2013

[32] Zhang, J., Wang, M., Hua, M., Yang, W., \& You, X.. Robust synchronization waveform design for massive IoT. 2017 IEEE Transactions on Wireless Communications, 16(11), 7551-7559.

[33] Silvestri, F., Acciarito, S., Cardarilli, G.C., Khanal, G.M., Di Nunzio, L., Fazzolari, R., Re, M. FPGA implementation of a low-power QRS extractor (2019) Lecture Notes in Electrical Engineering, 512, pp. 9 15

[34] G. C. Cardarilli, L. Di Nunzio R. Fazzolari, A. Nannarelli, M. Re Power Efficient Digital Front-End for Cognitive Radio Systems 2018 2018 52nd Asilomar Conference on Signals, Systems, and Computerspp $199-202$

[35] S. Acciarito, G.C. Cardarilli, L. Di Nunzio, R. Fazzolari, M. Re: wireless sensor node based on Microbial Fuel Cell Vol 409 Lecture Notes in Electrical Engineering pages 143-150 year 2017

[36] Khanal, G.M., Cardarilli, G., Chakraborty, A., Acciarito, S., Mulla, M.Y., Di Nunzio, L., Fazzolari, R., and Re, M. A ZnO-rGO composite thin film discrete memristor (2016) IEEE International Conference on Semiconductor Electronics, Proceedings, ICSE, 2016September, art. no. 7573608 , pp. $129-132$

[37] Khanal, G.M., Acciarito, S., Cardarilli, G.C., Chakraborty, A., Di Nunzio, L., Fazzolari, R., Cristini, A., M. Re, Susi, G. Synaptic behaviour in ZnO-rGO composites thin film memristor (2017) Electronics Letters, 53 (5), pp. 296-298.

[38] Acciarito, S., Cristini, A., Di Nunzio, L., Khanal, G.M., Susi, G. An a VLSI driving circuit for memristor-based STDP 12th Conference on Ph.D. Research in Microelectronics and Electronics, PRIME 2016, art. no. 7519503

[39] L. Bracciale, P. Loreti and G. Bianchi, The Sleepy Bird Catches More Worms: Revisiting Energy Efficient Neighbor Discovery (2016) IEEE Transactions on Mobile Computing, 15 (7), pp. 1812-1825.

[40] Cardarilli, G.C., Di Nunzio, L., Fazzolari, R., Re, M. TDES cryptography algorithm acceleration using a reconfigurable functional unit 2014 21st IEEE International Conference on Electronics, Circuits and Systems, ICECS 2014, art. no. 7050011, pp. 419-422.

[41] Cardarilli, G.C., Di Nunzio, L., Fazzolari, R., Pontarelli, S., Re, M., Salsano, A. Implementation of the AES algorithm using Reconfigurable Functional Unit (2011) ISSCS 2011 - International Symposium on Signals, Circuits and Systems, Proceedings, art. no. 5978668, pp. 97-100.

[42] Cardarilli, G.C., Di Nunzio, L., Fazzolari, R., Re, M., Silvestri, F. and Spanò, S. 2018, Energy consumption saving in embedded microprocessors using hardware accelerators, Telkomnika (Telecommunication Computing Electronics and Control), vol. 16, no. 3, pp. 1019-1026.

[43] D. Giardino, M. Matta and S. Spanò Energy Consumption Saving Using Hardware Accelerators in Image processing Electronics and Computer Science Scientific Reports Vol 1 No 1 Issue 1 (2019) 
[44] G. C. Cardarilli, L. Di Nunzio, R. Fazzolari, D. Giardino, M. Matta, M. Patetta, M. Re, S. Spanò Approximated compunting for low power Neural Networks (2019) Telkomnika (Telecommunication Computing Electronics and Control), 17 (3), ARTICLE IN PRESS

[45] D. Giardino, M. Matta, M. Re, F. Silvestri, S. Spanò IP Generator Tool for Efficient Hardware Acceleration of Self-Organizing Maps (2019) in: International Conference on Applications in Electronics Pervading Industry, Environment and Society. Springer, 2019

[46] G.C. Cardarilli, L. Di Nunzio, R. Fazzolari, D. Giardino, M. Matta, M. Re, F. Silvestri, S. Spanò Efficient Ensemble Machine Learning implementation on FPGA using Partial Reconfiguration (2019), ) in: International Conference on Applications in Electronics Pervading Industry, Environment and Society. Springer, 2019,

[47] Cardarilli, G. C., Cristini, A., Di Nunzio, L., Re, M., Salerno, M., \& Susi, G.. Spiking neural networks based on LIF with latency: Simulation and synchronization effects. 2013 IEEE Asilomar Conference on Signals, Systems and Computers, 1838-1842
[48] Susi, G., Toro, L. A., Canuet, L., López, M. E., Maestú, F., Mirasso, C. R., \& Pereda, E. (2018). A neuro-inspired system for online learning and recognition of parallel spike trains, based on spike latency, and heterosynaptic STDP. Frontiers in Neuroscience, 12

[49] M. Matta, G.C. Cardarilli, L. Di Nunzio, R. Fazzolari, D.Giardino, M. Re, F. Silvestri and S. Spanò Q-RTS: a Real-Time Swarm Intelligence based on Multi-Agent Q-Learning 2019 IET Electronics Letters ARTICLE IN PRESS

[50] Fallucchi, F., Zanzotto, F.M. Exploiting transitivity in probabilistic models for ontology learning (2012) Semi-Automatic Ontology Development: Processes and Resources, pp. 259-293.

[51] Fallucchi, F., Zanzotto, F.M. Transitivity in semantic relation learning Proceedings of the 6th International Conference on Natural Language Processing and Knowledge Engineering, NLP-KE 2010, art. no. 5587773. 